\title{
First Experience in Quality of Life Analysis After Bariatric Surgery
}

\author{
Arturs Ozolins*,**, Maris Pavars*, Janis Gardovskis*,** \\ *Department of General Surgery, Pauls Stradins University Hospital, Riga, Latvia \\ **Riga Stradins University, Latvia
}

\begin{abstract}
Summary
Introduction. The prevalence of obesity has increased markedly in the past 20 years therefore, bariatric surgery plays an important role in obesity treatment.

Aim of the study. The aim of this study was to evaluate the efficacy of Laparoscopic adjustable gastric banding (LAGB) and it influence on patients' quality of life (QOL) in the management of morbid obesity.

Materials and methods. 18 patients with morbid obesity underwent LAGB procedure at the Paul Stradins Clinical University Hospital, Riga, Latvia. Data collected were: age, sex, weight, height and operation time, body mass index (BMI), excessive body weight (EBW) and comorbidities. Patients fulfilled general Short Form Health Survey with 36 questions before operation (a), 6 month (b) and 12 month (c) after operation. License was acquired for SF-36v $2{ }^{\circledR}$ use in the study.

Results. The mean \pm SD age of patients was $42.5 \pm 11.8$ years $(25-63)$ with $83.3 \%$ of them female. The mean \pm SD weight was $133.7 \pm 20.3 \mathrm{~kg}(104-176)$, mean \pm SD height $168.7 \pm 9.4 \mathrm{~cm}(155-189)$ and mean BMl $46.2 \pm 6.6 \mathrm{~kg} / \mathrm{m} 2(36.8-58.8)$.

The mean \pm SD weight at 6 month follow up was $113.5 \pm 17.8 \mathrm{~kg}(\mathrm{p}=0.001)$. The postoperative mean BMI at 6 month follow up was $39.32 \pm 6.1 \mathrm{~kg} / \mathrm{m} 2(p=0.001)$. The mean percentage of excessive body weight (EBW) loss at 6 months was $30.4 \%$.

QOL life was higher after operation (b, c) comparing to preoperative data (a), but there were no statistically significant difference in any of the scales

Conclusions. The efficacy of LAGB surgery in long-term is still uncertain, but our early results are comparable to other series. To determine the true efficacy of LAGB in weight control and impact on QOL, a longer follow-up period is necessary, more cases to analyze and data from control group of general population.

Our findings suggest that life quality questionnaire SF-36v $2{ }^{\circledR}$ is good tool to measure patient's quality of life before and after operation, to discuss effectiveness of operations techniques and to observe quality of life dynamics.
\end{abstract}

Key words: laparoscopic adjustable gastric banding, LAGB, QOL, SF-36, obesity.

\section{INTRODUCTION}

The prevalence of obesity has increased markedly in the past 20 years, becoming a major public health issue (18, 19, 26). According to the World Health Organization, 'overweight' is defined as a body mass index (BMI), ie weight $(\mathrm{kg}) /[\text { height }(\mathrm{m})]^{2}$, of greater than $25 \mathrm{~kg} / \mathrm{m}^{2}$, whereas 'obese' is defined as a BMI of greater than $30 \mathrm{~kg} / \mathrm{m}^{2}(29)$. Obesity surgery is currently one of the most frequently performed surgical procedures in the USA and Europe with steadily increasing numbers (15). It is well-known that the higher one's BMI value, the higher his or her mortality wherewith many obesityrelated comorbidities have been documented $(2,8)$. The health risks of obesity include coronary artery disease, hypertension, diabetes, gallstones, cancer, orthopaedic problems, sleep apnoea and general debility (4).

Morbid obesity, defined as a BMI of greater than $35 \mathrm{~kg} /$ $\mathrm{m}^{2}$, occurs in $2 \%$ to $5 \%$ of the population of Europe, Australia, and the USA, and it is becoming increasingly common $(1,24)$. Despite nonsurgical treatment of obesity such as behavioral and pharmacologic methods have been proved to have an effect on short-term weight loss of approximately 5 to $10 \%$ of body weight (7), these methods are not very effective on long-term resolution of excesive body weight and its related comorbidities. Therefore, bariatric surgery plays an important role in obesity treatment $(6,12)$. Nowadays many surgeons have learned to perform this type of surgery laparoscopically rather than using an open approach (22). Currently LAGB and the laparoscopic Roux-en-Y gastric bypass are the most widely used methods for obesity treatment (16). LAGB which was introduced in early 1990s offers the advantages of minimally invasive surgery, adjustability, and reversibility. This purely gastric restrictive procedure involves the use of an adjustable silicone band placed around the gastric cardia, which creates a small gastric pouch $(15 \mathrm{~mL})$ with a narrow outlet. We used Ethicon Endo-Surgery LGB 12 ${ }^{\mathrm{TM}}$ Adjustable Gastric Band and Gastric Calibration Tube GCT-360.

To date, comparative studies are mainly looking at clinical data of weight loss, change of comorbidities and morbidity (27), but only few comparative studies on health-related quality of life exist $(10,28)$.

QOL is relevant parameter in evaluation of surgical treatment because it perfectly shows physical as well as mental health of the patient and it is one of the most important criteria to judge about patient's well being after operation. 


\section{AIM OF THE STUDY}

The aim of this study was to evaluate the efficacy of laparoscopic adjustable gastric banding and it influence on patients QOL in the management of morbid obesity.

\section{MATERIALS AND METHODS}

Between 1 January 2008 and 1 May 2009, a total of 18 patients with morbid obesity underwent LAGB procedure at the Paul Stradins Clinical University Hospital, Riga, Latvia. Patients were considered as candidates for operation if the following conditions were met: aged between 18 and 65 years, BMI of greater than $40 \mathrm{~kg} / \mathrm{m}^{2}$ or greater than $35 \mathrm{~kg} / \mathrm{m}^{2}$ with serious comorbidities, no excessive intake of sweets or alcohol. All patients had history of obesity for more than 5 years and had failed conservative management such as diet, behavioral therapy, and pharmacotherapy in the past. Obesity-related comorbidities was present in 55.5\% of patients, which included 3 patients with diabetes mellitus (DM), 6 patients with hypertension, 6 patients with joint and spine pain, and 1 patient with dyspnoea. Exclusion criteria included previous gastric surgery, a large hiatal hernia, and a history of alcohol addiction and poorly controlled non-obesity-related medical diseases.

This study was approved by the Ethics Committee of Paul Stradins Clinical University Hospital. Written consent was given by all patients.

Operative procedure. A high-dose low-molecular-weight heparin was given in the morning of operation, and an antibiotic prophylaxis was given during induction. The operation was performed under general anaesthesia. The patient was in the reverse Trendelenburg position with the legs extended in stirrup, and the operating surgeon was standing between the patient's legs. A five-trocar technique was used with a 15-mm port to allow insertion of the gastric band into the peritoneal cavity. After the liver was retracted with laparoscopic retractor, the pars flaccida technique was performed (30). The dissection began at the base of the left crus well above the first short gastric artery to free the angle of the His of the stomach. Following the opening of the left phrenoesophageal ligament, the gastro-hepatic ligament (pars flaccida) was divided, and blunt dissection was performed to create a passage between the diaphragmatic pillars and the posterior aspect of the gastro-oesophageal junction. The empty band was then inserted through the $15-\mathrm{mm}$ trocar and pulled through the retrogastric tunnel with the help of the Goldfinger dissector. Once the band was locked in place, the device was anchored anteriorly by three gastrogastric stitches with non-absorbable sutures. The fundus of the stomach below the band was stitched to the gastroesophageal junction completely covering the anterior aspect of the band to prevent herniation of the fundus. After the band was secured, a separate incision was made just above the xiphoid, and the port was connected and sutured subcutaneously to the presternal fascia (29). In the postoperative course, all the patients received a contrast study of the esophagus and stomach 1 day after procedure. The patients were discharged as soon as sufficient oral fluid and soft food intake was possible.

For all patients, the data collected were age, sex, weight, height and operation time, BMI, excessive body weight (EBW) and comorbidities.

At two points in time, patients were weighed, and BMI was calculated. The first measurement was taken before surgery and the second 6 month after operation.

Patients fulfilled general SF-36v2® Short Form Health Survey with 36 questions before operation (a), 6 month (b) and 12 month (c) after operation. License was acquired for SF-36v2 @ health survey questionnaire use in the study.

Questionnaire consists of internationally accepted SF-36v2 @ health research protocol which set so that minimum psychometrical standard parameters which are not specific neither to age nor illness or treatment method where included (9). Acquired data entered into the data base and statistical processing was made. After data processing eight health concepts is possible to measure: physical functioning - PF, role physical - RP, bodily pain - BP, general health - GH, vitality - VT, social functioning - SF, role emotional - RE, mental health $\mathrm{MH}$. For scales the highest possible score of 100 is achieved when no limitations or disabilities are observed. Missing values in the SF-36v2 ${ }^{\circledR}$ were replaced by mean individual scores, as recommended in the manual.

3 health scales (PF, RP, and BP) with statistical significance measure physical health but each of them describes different aspects: physical functioning (PF) measure limitation in physical activities; role physical (RP) - inability to do everyday activities due to physical problems; bodily pain (BP) focuses on physical pain.

Mental component consist of MH, RE and SF parameters: social functioning (SF) and role emotional (RE) shows limitations due to personal or emotional problems; mental health $(\mathrm{MH})$ indicates psychological stress symptoms.

General health (GH) and vitality (VT) scales are sensitive on physical and mental health. Vitality (VT) characterises tiredness or joy of living.

Twelve month after operation patients answered also to specific questions related to operation result.

For acquiring data and further processing ABBYY FormReader 6.5, QualityMetric Health Outcomes ${ }^{\mathrm{TM}}$ Scoring Software 2.0, SPSS 16.0 and Microsoft Excel programs were used. For analysis, the presence of the questionnaires at the three points in time was required. For analysis, the nonparametric Friedman K-related samples test was used, as variables were not normally distributed. Statistical significance was defined as $p<0.05$. To analyze QOL between 2 surveys we used Wilcoxon Signed Ranks test.

\section{RESULTS}

Eighteen patients were enrolled in the study. The mean \pm SD age of patient was $42.5 \pm 11.8$ years (25-63) with $83.3 \%$ of them female. The mean \pm SD weight was $133.7 \pm 20.3 \mathrm{~kg}$ (104-176), mean \pm SD height $168.7 \pm$ $9.4 \mathrm{~cm}$ (155-189) and mean BMI $46.2 \pm 6.6 \mathrm{~kg} / \mathrm{m}^{2}(36.8-$ 
58.8). The demographic and clinical characteristics of 18 patients with morbid obesity are summarized in Table 1.

Median operating time was 100 minutes (80-120 minutes) and all cases were successfully performed without open conversion. The median hospital stays 3 days (2-4 days).

The mean \pm SD weight at 6 month follow up was $113.5 \pm$ $17.8 \mathrm{~kg}(\mathrm{p}=0.001)$. The postoperative mean BMI at 6 month follow up was $39.32 \pm 6.1 \mathrm{~kg} / \mathrm{m}^{2}(\mathrm{p}=0.001)$. The mean percentage of excessive body weight (EBW) loss [\% EBW loss=weight loss/(initial body weight-ideal body weight at BMI 25) x 100\%] at 6 months was $30.4 \%$. The results are summarized in Table 2 .

There were no intraoperative complications and no blood transfusion was needed. We analyzed quality of life using SF-36v2 ® Short Form Health Survey before operation (a), 6 weeks (b) and 6 month after operation (c).

To analyze QOL dynamics we used Friedman test and evidently QOL life was higher after operation (b, c) comparing to preoperative data (a), but there were no statistically significant difference in any of the scales (Figure 1).

Using Wilcoxon Signed Ranks test we analyzed QOL between 2 surveys: $c-a, c-b, b-a$, and there were no statistically significant improvement in any of the scales.

With the center-specific questionnaire 12 month after operation, the patients were asked how satisfied they were with results of the operation. Of the patients, $100 \%$ stated to be satisfied with the operation. However two from five patients wish to have greater weight reduction. All patients reported to have increase in their QOL and could recommend this type of operation to others.

\section{DISCUSSION}

Obesity is becoming a worldwide phenomenon. Since the first jejuno-ileal bypass performed by Kremer and Linner in 1960s, various approaches and procedures have been developed to deal with obesity. Surgical treatment has had an established role in the management of morbid obesity. The criteria for selecting patients for bariatric procedures are based mainly on the BMI definition of obesity as recommended by the World Health Organization.

Numerous comparative studies for the treatment of morbid obesity have been done, but the bariatric procedure of choice is still under debate.

Malabsorptive procedures, such as jejuno-ileal, biliopancreatic, or gastric bypass allow the patient to 'lose weight while eating' (20). The Roux-en-Y gastric bypass has recently been adapted for use with laparoscopy, achieving an acceptably low rate of complications (3.3\% major and $27 \%$ minor) and an excellent weight loss of at least $60 \%$ EBW (21). However, this procedure is both difficult and lengthy ( 2 to 3 times longer than our procedure), and complications, such as anastomotic failure and intestinal obstruction, are potentially lethal. Restrictive procedures like LAGB induce slow gastric emptying via a small pouch and create early satiation allowing a $40 \%$ to $50 \%$ reduction in the excess weight in 1 to 2 years (17).

An adjustable gastric banding (AGB) device which was introduced by Kuzmak (11) has been adopted and nowadays is widely used in Europe, Australia, and the US. Main advantages of AGB include its simplicity, reversibility, the adjustability of the band's stoma size. In the medical literature, the SF-36 questionnaire is widely used and well validated in all types of studies as well as in the obesity literature (3). Several studies suggest that quality of life is improved by bariatric procedures in general and that bypass affects the clinical aspects of weight loss and comorbidities more positively than the banding procedure $(13,25$, and 27$)$.

In comparative study of laparoscopic banding vs Rouxen-Y gastric bypass Muller et al. assessed the QOL using SF-36 and Moorehead-Ardelt II (MA II) questionnaire and found that patients after laparoscopic gastric bypass and laparoscopic gastric banding have a "good" quality of life, as measured by the MA II. This finding was reinforced by the SF 36 that also demonstrated postoperative quality of life scoring similar to normal values. Quality of life indexes were not different between the two operation procedures, as long as the banding procedure was successful without conversion to another procedure in the long term (15). Sears et al. in his study has shown that significant weight loss is achieved after gastric bypass surgery and that there is a significant improvement in both quality of life scores and obesity-related conditions (23). Mui et al. in prospective study evaluated the outcome of intragastric balloon (IGB) on weight loss and the impact of it on obesityrelated illnesses and quality of life. He was able to show that IGB improves seven out of eight domains and both physical and mental component of quality of life of SF36. It is a very valid option for those who do not opt for or not suitable for bariatric surgery (14). Chang et al. in his study included healthy control subjects and found that QOL for patients with morbid obesity in Taiwan is worse than for those of general population and if BMI level is above 32 then lower are the domain scores (4). In the past decade, the LAGB technique has improved with an estimated 100000 patients having had this procedure (5). The LAGB procedure coupled with the increased experience of the surgeon seems to minimize the chances of complication.

In our study of the LAGB for the treatment of morbid obesity we analyzed QOL outcomes as well as weight reduction. In our series, we achieved a mean excessive weight loss of $30.4 \%$ in the 6 month period which is comparable to results published in literature. 6 month and 12 month after operation compared to preoperative data, QOL improved in all the scales of SF-36v2 ${ }^{8}$ but it was not statistically significant due to small number of cases.

To determine the true efficacy of LAGB in weight control and impact on QOL, a longer follow-up period is necessary, more cases to analyze and data from control group of general population. 


\section{CONCLUSIONS}

For morbidly obese patients weight reduction surgery is the only proven method for long-term weight control. The efficacy of LAGB surgery in long-term is still uncertain, but our early results are comparable to other series. The laparoscopic approach allows early mobilisation, short hospital stay, early return to work, and fewer wound complications. This reversible and adaptable procedure promises to be a good alternative to other bariatric surgeries, provided that the morbidity rate remains low.

Multidisciplinary team consisting of physician, nutritionist, surgeon, together with the psychologist is essential in treatment of obese patients.

Our findings suggest that life quality questionnaire SF-36v2 $®$ is good tool to measure patient's quality of life before and after operation, to discuss effectiveness of operations techniques and to observe quality of life dynamics.

\section{Conflict of interest: None}

\section{REFERENCES}

1. Abraham S, Johnson CL. Prevalence of severe obesity in adults in the United States // Am J Clin Nutr, 1980; 33(2 Suppl):364 - 9

2. Adams KF, et al. Overweight, obesity, and mortality in a large prospective cohort of persons 50 to 71 years old // N Engl J Med, 2006; 355(8):763 - 78

3. Ballantyne GH. Measuring outcomes following bariatric surgery: weight loss parameters, improvement in co-morbid conditions, change in quality of life and patient satisfaction // Obes Surg, 2003; 13(6):954 - 64

4. Chang CY, et al. Health-related Quality of Life in Adult Patients with Morbid Obesity Coming for Bariatric Surgery // Obes Surg, 2008; [Epub ahead of print]

5. Cottam DR, Mattar SG, Schauer PR. Laparoscopic era of operations for morbid obesity // Arch Surg, 2003; 138(4):367 - 75

6. Davis MM, et al. National trends in bariatric surgery 1996-2002 // Arch Surg, 2006; 141(1):71 - 4; discussion 75

7. Delinsky SS, Latner JD, Wilson GT. Binge eating and weight loss in a self-help behavior modification program//Obesity (SilverSpring), 2006; 14(7):12449

8. Flegal KM, et al. Excess deaths associated with underweight, overweight, and obesity // JAMA, 2005; 293(15):1861 - 7

9. Guyatt GH, Feeny DH, Patrick DL. Measuring health-related quality of life // Ann Intern Med, 1993; $118(8): 622-9$

10. Hell E, et al. Evaluation of health status and quality of life after bariatric surgery: comparison of standard Roux-en-Y gastric bypass, vertical banded gastroplasty and laparoscopic adjustable silicone gastric banding // Obes Surg, 2000; 10(3):214 - 9
11. Kuzmak LI. A Review of Seven Years' Experience with Silicone Gastric Banding // Obes Surg, 1991; $1(4): 403-408$

12. Maggard MA, et al. Meta-analysis: surgical treatment of obesity // Ann Intern Med, 2005; 142(7):547 59

13. Mognol P, Chosidow D, Marmuse JP. Laparoscopic gastric bypass versus laparoscopic adjustable gastric banding in the super-obese: a comparative study of 290 patients // Obes Surg, 2005; 15(1):76-81

14. Mui WL, et al. Impact on Obesity-Related Illnesses and Quality of Life Following Intragastric Balloon // Obes Surg, 2008; [Epub ahead of print]

15. Muller MK, et al. Quality of life after bariatric surgery-a comparative study of laparoscopic banding vs. bypass // Obes Surg, 2008; 18(12):1551 7

16. Nguyen NT, et al. Result of a national audit of bariatric surgery performed at academic centers: a 2004 University HealthSystem Consortium Benchmarking Project // Arch Surg, 2006; 141(5):445 - 9; discussion 449 - 50

17. Nightengale ML, et al. Prospective evaluation of vertical banded gastroplasty as the primary operation for morbid obesity // Mayo Clin Proc, $1991 ; 66(8): 773-82$

18. Ogden CL, et al. Prevalence of overweight and obesity in the United States, 1999-2004 // Jama, 2006; 295(13):1549 - 55

19. Prentice AM. The emerging epidemic of obesity in developing countries // Int J Epidemiol, 2006; 35(1):93-9

20. Requarth JA, et al. Long-term morbidity following jejunoileal bypass. The continuing potential need for surgical reversal // Arch Surg, 1995; 130(3):318 25

21. Schauer PR, et al. Outcomes after laparoscopic Roux-en-Y gastric bypass for morbid obesity // Ann Surg, 2000; 232(4):515-29

22. Schirmer B. Laparoscopic bariatric surgery // Surg Endosc, 2006; 20 Suppl 2:450 - 5

23. Sears D, et al. Evaluation of gastric bypass patients 1 year after surgery: changes in quality of life and obesity-related conditions // Obes Surg, 2008; $18(12): 1522-5$

24. Seidell JC. Obesity in Europe: scaling an epidemic // Int J Obes Relat Metab Disord, 1995; 19 Suppl 3: $\mathrm{S} 1-4$

25. Sjostrom L, et al. Lifestyle, diabetes, and cardiovascular risk factors 10 years after bariatric surgery // N Engl J Med, 2004; 35 1(26):2683 - 93

26. Sturm R. Increases in clinically severe obesity in the United States, 1986-2000 // Arch Intern Med, 2003; 163(18):2146-8

27. Weber $M$, et al. Laparoscopic gastric bypass is superior to laparoscopic gastric banding for treatment of morbid obesity // Ann Surg, 2004; 240(6):975 - 82; discussion 982 - 3

28. Wolf AM, et al. BAROS: an effective system to evaluate the results of patients after bariatric 
surgery // Obes Surg, 2000; 10(5):445 - 50

29. Wong SK, et al. Laparoscopic adjustable gastric banding for the treatment of morbidly obese patients: early outcome in a Chinese cohort // Hong Kong Med J, 2005; 11 (1):20 - 9

30. Zinzindohoue $\mathrm{F}$, et al. Laparoscopic gastric banding: a minimally invasive surgical treatment for morbid obesity: prospective study of 500 consecutive patients // Ann Surg, 2003; 237(1):1 - 9

\section{Address:}

Arturs Ozolins,

Pauls Stradins Clinical University Hospital Department of Surgery

Pilsonu street 13, Riga, LV-1002

E-mail: archa_oz@inbox.lv
Table 1. Demographic and clinical characteristics of patients

\begin{tabular}{|c|c|}
\hline Total no. of patients & 18 \\
\hline Age $^{\mathrm{a}}$ (year) & $42.5 \pm 11.8$ \\
\hline Gender $(\mathrm{F}: \mathrm{M})$ & $15 / 3$ \\
\hline Weight $^{\mathrm{a}}(\mathrm{kg})$ & $133.7 \pm 20.3$ \\
\hline Height $^{\mathrm{a}}(\mathrm{cm})$ & $168.7 \pm 9.4$ \\
\hline BMI $^{\mathrm{a}}\left(\mathrm{kg} / \mathrm{m}^{2}\right)$ & $46.2 \pm 6.6$ \\
\hline \% with comorbidities & 55.5 \\
\hline
\end{tabular}

a Mean \pm SD

Table 2. Weight reduction results

\begin{tabular}{|c|c|c|c|}
\hline & $\begin{array}{c}\text { Before LAGB } \\
\mathrm{n}=18\end{array}$ & $\begin{array}{c}\text { After LAGB } \\
(6 \text { month), } \\
\mathrm{n}=18\end{array}$ & $p$ value \\
\hline Weight $^{\mathrm{a}}(\mathrm{kg})$ & $133.7 \pm 20.3$ & $113.5 \pm 17.8$ & $0.001^{\mathrm{b}}$ \\
\hline BMI $^{\mathrm{a}}\left(\mathrm{kg} / \mathrm{m}^{2}\right)$ & $46.2 \pm 6.6$ & $39.32 \pm 6.1$ & $0.001^{\mathrm{b}}$ \\
\hline EBW loss $(\%)$ & & 30.4 & \\
\hline
\end{tabular}

${ }^{\text {a }}$ Mean \pm SD

${ }^{\mathrm{b}}$ Statistically significant
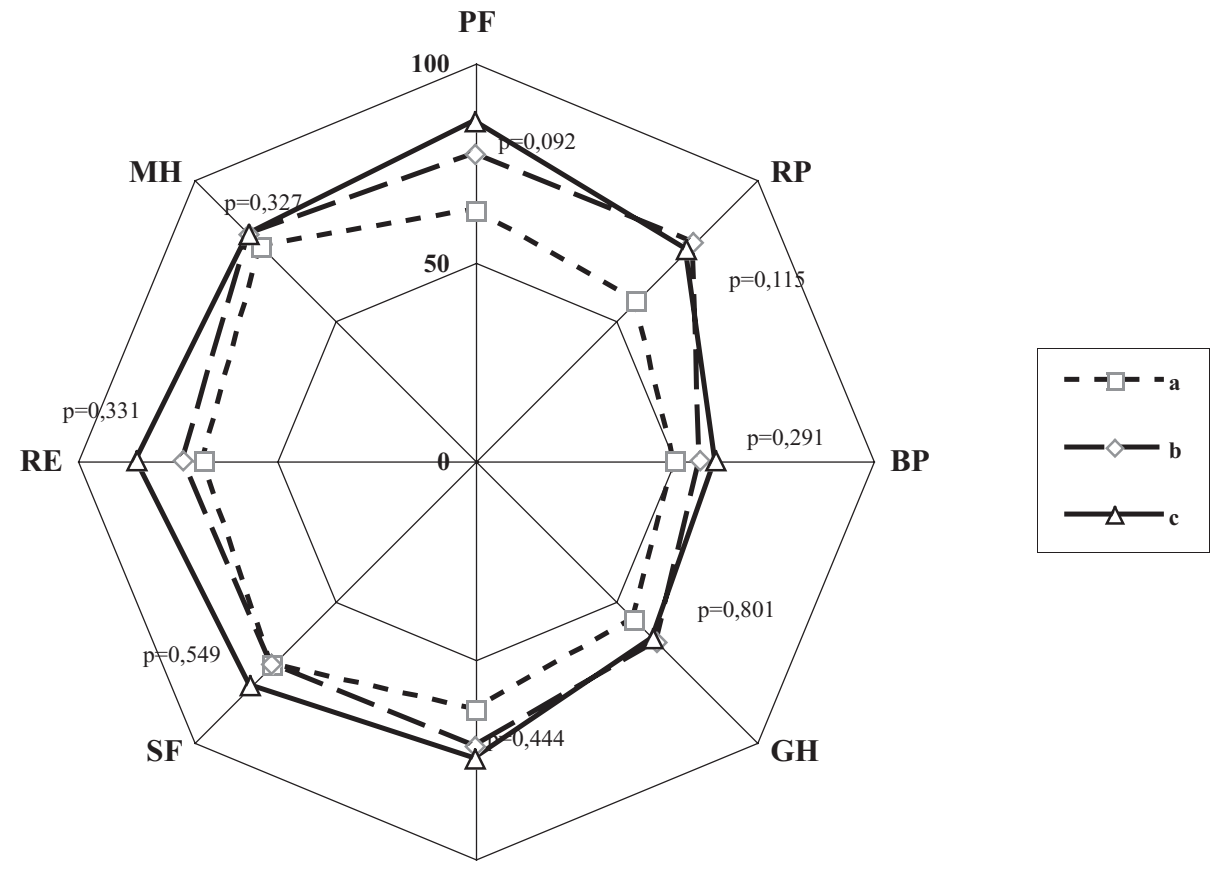

VT

\begin{tabular}{|c|c|c|c|c|c|c|c|c|}
\hline & PF & RP & BP & GH & VT & SF & RE & MH \\
\hline $\mathbf{a}$ & 63.00 & 57.08 & 50.00 & 56.40 & 62.50 & 72.50 & 68.33 & 76.00 \\
\hline $\mathbf{b}$ & 77.00 & 77.50 & 56.60 & 64.55 & 71.87 & 72.50 & 73.33 & 80.50 \\
\hline $\mathbf{c}$ & 85.14 & 75.00 & 60.40 & 63.15 & 75.00 & 80.00 & 85.00 & 80.25 \\
\hline $\mathbf{X}^{\mathbf{2}}$ & 4.778 & 4.333 & 2.471 & 0.444 & 1.625 & 1.200 & 2.211 & 2.235 \\
\hline $\mathbf{p}^{\mathbf{a}}$ & $\mathbf{0 . 0 9 2}$ & $\mathbf{0 . 1 1 5}$ & $\mathbf{0 . 2 9 1}$ & $\mathbf{0 . 8 0 1}$ & $\mathbf{0 . 4 4 4}$ & $\mathbf{0 . 5 4 9}$ & $\mathbf{0 . 3 3 1}$ & $\mathbf{0 . 3 2 7}$ \\
\hline
\end{tabular}

${ }^{\mathrm{a}}$ Friedman test

Fig 1. Preoperative, 6 month and 12 month postoperative SF-36v2 ${ }^{\circledR}$ QOL dynamics 